\title{
Neumann problem in a disk for fourth-order improperly elliptic equations
}

\author{
Kateryna O. Buryachenko, Yuliya Kudrych
}

(Presented by I. I. Skrypnik)

\begin{abstract}
We have established and investigated the sufficient conditions of solvability of the Neumann problem for all classes of fourthorder improperly elliptic equations in a unit disk $\mathrm{K}$ in the space $C^{4}(K) \cap$ $C^{3, \alpha}(K)$.
\end{abstract}

2010 MSC. 35J40.

Key words and phrases. Fourth-order partial differential equations, Neumann problem, Dirichlet problem, kernel, properly and improperly elliptic equations, Fredholm problem.

\section{Introduction}

This paper is devoted to the existence of a solution of the Neumann problem in a disk for all classes of fourth-order improperly elliptic differential equations. This problem belongs to fairly important questions of the correctness of the so-called general boundary value problems for highorder differential equations, which spring from the works by Hormander and Wischik, who, with the help of extension theory, proved the existence of a correct boundary problem for linear differential equations of arbitrary order with constant complex coefficients in a bounded domain with a smooth boundary. (Note that this theory obtained its present day development in the works by Grubb [1] and Hormander [2]). Later, the question of the correctness of boundary value problems for various types of differential equations was studied by numerous researchers. Studies of the Dirichlet problem for second order improperly elliptic differential equations began from the work by Bitsadze [3], who constructed an example of second order elliptic equation for which the homogeneous Dirichlet problem in a disk has a countable set of linearly independent solutions. The question of the correctness of boundary value problems is tightly

Received 25.07.2017

ISSN 1810 - 3200. (C) Інститут прикладної математики і механіки НАН України 
bound with investigations of the spectrum of operator of the corresponding problem. As to the works in this direction, we should mention the recent publications of Albeverio [4], Brown [5] et al. For high order equations, in particular, for fourth order equations and later for equations of arbitrary even order $2 m, m \geq 2$, the Dirichlet problem was studied by Buryachenko [6-8]. As to the Neumann problem, some conditions of its solvability in a disk for second order equations containing the lowest part were obtained by Bonanno and Pizzimenti [9]. For equations of general form with a homogeneous symbol of the fourth order and higher, the Neumann problem has been investigated only for the properly elliptic equation (see [7]). In the present work, we propose to carry over the methods of investigations of Babayan and Buryachenko, which were applied for studying the Dirichlet [6] and properly elliptic Neumann problem [7] for the case of all classes of improperly elliptic Neumann problem. In the work [13] we have already succeeded in performing this and, as a result, obtained the sufficient conditions of existence of the classical solution of the Neumann problem but only for one class of improperly elliptic fourth order equations in the space $C^{4}(K) \cap C^{3, \alpha}(K)$. Here we extend previous result and obtain sufficient conditions for all classes of improperly elliptic fourth order equations.

\section{Formulation of the Problem}

Consider the Neumann problem in a disk $K \subset R^{2}$ for fourth-order improperly elliptic equations with constant complex coefficients:

$$
\begin{gathered}
L\left(\partial_{x}\right) u=a_{0} \frac{\partial^{4} u}{\partial x_{1}^{4}}+a_{1} \frac{\partial^{4} u}{\partial x_{1}^{3} \partial x_{2}}+a_{2} \frac{\partial^{4} u}{\partial x_{1}^{2} \partial x_{2}^{2}}+a_{3} \frac{\partial^{4} u}{\partial x_{1} \partial x_{2}^{3}}+a_{4} \frac{\partial^{4} u}{\partial x_{2}^{4}}=0, \\
\left.\frac{\partial^{2} u}{\partial \nu^{2}}\right|_{\partial K}=f_{1},\left.\frac{\partial^{3} u}{\partial \nu^{3}}\right|_{\partial K}=f_{2},
\end{gathered}
$$

where $\vec{\nu}$ is the unit vector of an external normal, $\partial_{x}=\left(\frac{\partial}{\partial x_{1}}, \frac{\partial}{\partial x_{2}}\right), a_{k} \in$ $C, k=0,1, \ldots, 4$, and $f_{1} \in C^{1, \alpha}(\partial K), f_{2} \in C^{\alpha}(\partial K)$ are functions specified on the boundary $\partial K, 0<\alpha<1$, which can be prolonged to analytic functions in the disk $\mathrm{K}$ and outside it. We recall the important definition, which is used below.

Definition 1. Let $\lambda_{j}$ be the roots of the characteristic polynomial $L(1, \lambda)=a_{0} \lambda^{4}+a_{1} \lambda^{3}+a_{2} \lambda^{2}+a_{3} \lambda+a_{4}=0$. We assume them to be complex, $\lambda_{j} \in C$, i.e., Eq. (1) is elliptic. The elliptic equation (1) is called properly elliptic if the roots $\lambda_{j}$ are situated equally in the positive 
and negative imaginary planes:

$$
\begin{gathered}
\operatorname{Im} \lambda_{\mathrm{j}}>0, \mathrm{j}=1,2, \\
\operatorname{Im} \lambda_{\mathrm{k}}<0, \mathrm{k}=3,4 .
\end{gathered}
$$

As an example of a second order properly elliptic equation, we can mention the Laplace equation

$$
\Delta u=0, \lambda_{1}=i, \lambda_{2}=-i .
$$

As examples of improperly elliptic equations, we can mention the famous Bitsadze's examples ([3]):

$$
\left(\frac{\partial}{\partial x_{1}}+i \frac{\partial}{\partial x_{2}}\right)^{2} u=\frac{\partial^{2} u}{\partial x_{1}^{2}}+2 i \frac{\partial^{2} u}{\partial x_{1} \partial x_{2}}-\frac{\partial^{2} u}{\partial x_{2}^{2}}=0
$$

and

$$
\left(\frac{\partial}{\partial x_{1}}-i \frac{\partial}{\partial x_{2}}\right)^{2} u=\frac{\partial^{2} u}{\partial x_{1}^{2}}-2 i \frac{\partial^{2} u}{\partial x_{1} \partial x_{2}}-\frac{\partial^{2} u}{\partial x_{2}^{2}}=0 .
$$

Further it has been proved (see [10]) that each arbitrary order $2 m$, $m \geq 2$ elliptic equation is improperly elliptic equation, if all differences $\varphi_{i}-\varphi_{j}, i, j=1,2, \ldots, 2 m$ are real. Here $\varphi_{j}$ is solution of the equation $-\tan \varphi_{j}=\lambda_{j}$, for each $j=1,2, \ldots, 2 m$.

\section{Improperly elliptic equations in general position}

Assume that all roots of the characteristic equation are simple and not equal to $\pm i$. Obviously, for fourth order equations, one can single out the following two classes of improperly elliptic equations:

1.

$$
\operatorname{Im} \lambda_{1}>0, \operatorname{Im} \lambda_{2}>0, \operatorname{Im} \lambda_{3}>0, \operatorname{Im} \lambda_{4}<0,
$$

2 .

$$
\operatorname{Im} \lambda_{1}>0, \operatorname{Im} \lambda_{2}>0, \operatorname{Im} \lambda_{3}>0, \operatorname{Im} \lambda_{4}>0 .
$$

The higher the order of the equations, the more such classes can be singled out: for equations of arbitrary even order $2 \mathrm{~m}$, the number of such classes is $\mathrm{m}$.

Hence, we assume Eq. (1) to be a improperly elliptic equation of general form, i.e., the roots $\lambda_{1}, \lambda_{2}, \lambda_{3}, \lambda_{4}$ of its characteristic equation satisfy (3) or (4).

Let us study the question of solvability the Neumann boundary value problem (1), (2) for each of these classes. The basic method of our study is the method of the representation of analytic functions proposed by Tovmasyan [11]. For using this method, we rewrite problem (1), (2) in complex variables. 


\subsection{Class (3) of Improperly Elliptic Equations}

We introduce complex numbers $\mu_{k}$ constructed from the roots $\lambda_{k}, k=$ $1, \ldots, 4$ of the characteristic equation as follows:

$$
\mu_{1}=-\frac{\lambda_{1}-i}{\lambda_{1}+i}, \mu_{2}=-\frac{\lambda_{2}-i}{\lambda_{2}+i}, \mu_{3}=-\frac{\lambda_{3}-i}{\lambda_{3}+i}, \mu_{4}=-\frac{\lambda_{4}+i}{\lambda_{4}-i} .
$$

Let us rewrite problem (1), (2) in complex variables. We take

$$
x=\frac{z+\bar{z}}{2}, y=\frac{z+\bar{z}}{2 i}
$$

and then

$$
\frac{\partial}{\partial z}=\frac{1}{2}\left(\frac{\partial}{\partial x}-i \frac{\partial}{\partial y}\right), \frac{\partial}{\partial \bar{z}}=\frac{1}{2}\left(\frac{\partial}{\partial x}+i \frac{\partial}{\partial y}\right) .
$$

Applying these expressions to the derivatives that enter into (1), we obtain the new form of this equation:

$$
\left(\frac{\partial}{\partial z}-\mu_{1} \frac{\partial}{\partial \bar{z}}\right)\left(\frac{\partial}{\partial z}-\mu_{2} \frac{\partial}{\partial \bar{z}}\right)\left(\frac{\partial}{\partial z}-\mu_{3} \frac{\partial}{\partial \bar{z}}\right)\left(\frac{\partial}{\partial \bar{z}}-\mu_{4} \frac{\partial}{\partial z}\right) u(z)=0 .
$$

We also rewrite the Neumann boundary conditions (2) in complex variables. For this purpose, we use the relations

$$
\left.\frac{\partial u}{\partial \nu}\right|_{\partial K}=z \frac{\partial u}{\partial z}+\bar{z} \frac{\partial u}{\partial \bar{z}},\left.\quad \frac{\partial^{m} u}{\partial \nu^{m}}\right|_{\partial K}=\left(z \frac{\partial u}{\partial z}+\bar{z} \frac{\partial u}{\partial \bar{z}}\right)^{m}, m=2,3 .
$$

Finally, we have the following boundary conditions:

$$
\begin{aligned}
& \left.\left(z^{2} \frac{\partial^{3} u}{\partial z^{3}}+2 z \bar{z} \frac{\partial^{3} u}{\partial z^{2} \partial \bar{z}}+\bar{z}^{2} \frac{\partial^{3} u}{\partial \bar{z}^{2} \partial z}+2 z \frac{\partial^{2} u}{\partial z^{2}}+2 \bar{z} \frac{\partial^{2} u}{\partial z \partial \bar{z}}\right)\right|_{\partial K}=F_{1}(z), \\
& \left.\left(\bar{z}^{2} \frac{\partial^{3} u}{\partial \bar{z}^{3}}+2 z \bar{z} \frac{\partial^{3} u}{\partial z \partial \bar{z}^{2}}+z^{2} \frac{\partial^{3} u}{\partial z^{2} \partial \bar{z}}+2 \bar{z} \frac{\partial^{2} u}{\partial \bar{z}^{2}}+2 z \frac{\partial^{2} u}{\partial z \partial \bar{z}}\right)\right|_{\partial K}=F_{2}(z)
\end{aligned}
$$

Here, $F_{1}(z)$ and $F_{2}(z)$ are functions constructed with the help of specified functions $f_{1} \in C^{1, \alpha}(\partial K)$ and $f_{2} \in C^{\alpha}(\partial K)$ from the Neumann conditions (2). 


\subsection{Solvability of Neumann problem (5), (6), (7) under condition (3)}

Note that the condition of improper ellipticity of Eq. (1) leads (with regard for formulas (3) and (5)) to the conditions $\left|\mu_{k}\right|<1, k=1,2,3,4$. Obviously, the solution $\mathrm{u}(\mathrm{z})$ of Eq. (5) will have the following form:

$$
u(z)=\Phi_{1}\left(z+\mu_{1} \bar{z}\right)+\Phi_{2}\left(z+\mu_{2} \bar{z}\right)+\Phi_{3}\left(z+\mu_{3} \bar{z}\right)+\Phi_{4}\left(\bar{z}+\mu_{4} z\right),
$$

where $\Phi_{k}, k=1,2,3,4$ are certain functions fairly smooth and analytic together with their derivatives. We find them from the boundary conditions (6), (7). The principal role in this study will belong to lemma on the representation of analytic functions.

Lemma 1. [11] Suppose that $\mu, \nu$ are complex numbers such that: $|\mu|<1,|\nu|<1, \zeta_{1}: K \rightarrow D_{1}, \zeta_{1}=z+\mu \bar{z}, \zeta_{2}: K \rightarrow D_{2}, \zeta_{2}=\bar{z}+\nu z$ are certain conformal mappings of the unit disk $K$ in domains $D_{1}$ and $D_{2}$, respectively. Further, let $\varphi\left(\zeta_{1}\right)$ and $\psi\left(\zeta_{2}\right)$ be functions analytic and continuous in $D_{1}$ and $D_{2}$, respectively. Then

$$
\varphi(z+\mu \bar{z})=\omega(z)+\omega(\mu \bar{z}), \psi(\bar{z}+\nu z)=\rho(\bar{z})+\rho(\nu z),
$$

where $\omega$ and $\rho$ are analytic functions in the unit disk $K$.

We assume the functions $\Phi_{k}^{\prime \prime}, k=1,2,3,4$ to be analytic and continuous in the corresponding domains of their definition. Applying Lemma 1 to them, we obtain

$$
\begin{aligned}
& \Phi_{1}^{\prime \prime}\left(z+\mu_{1} \bar{z}\right)=\omega(z)+\omega\left(\mu_{1} \bar{z}\right), \Phi_{2}^{\prime \prime}\left(z+\mu_{2} \bar{z}\right)=\gamma(z)+\gamma\left(\mu_{2} \bar{z}\right), \\
& \Phi_{3}^{\prime \prime}\left(z+\mu_{3} \bar{z}\right)=\alpha(z)+\alpha\left(\mu_{3} \bar{z}\right), \Phi_{4}^{\prime \prime}\left(\bar{z}+\mu_{4} z\right)=\beta(\bar{z})+\beta\left(\mu_{4} z\right) .
\end{aligned}
$$

Here, $\alpha, \beta, \gamma, \omega$ are certain analytic functions in the unit disk, and, hence, we can expand them in power series:

$$
\begin{aligned}
& \omega(z)=\sum_{k=1}^{\infty} A_{k} z^{k}, \omega\left(\mu_{1} \bar{z}\right)=\sum_{k=1}^{\infty} A_{k}\left(\mu_{1} \bar{z}\right)^{k}, \\
& \gamma(z)=\sum_{k=1}^{\infty} B_{k} z^{k}, \gamma\left(\mu_{2} \bar{z}\right)=\sum_{k=1}^{\infty} B_{k}\left(\mu_{2} \bar{z}\right)^{k}, \\
& \alpha(z)=\sum_{k=1}^{\infty} C_{k} z^{k}, \alpha\left(\mu_{3} \bar{z}\right)=\sum_{k=1}^{\infty} C_{k}\left(\mu_{3} \bar{z}\right)^{k}, \\
& \beta(\bar{z})=\sum_{k=1}^{\infty} D_{k} \bar{z}^{k}, \beta\left(\mu_{4} z\right)=\sum_{k=1}^{\infty} D_{k}\left(\mu_{4} z\right)^{k} .
\end{aligned}
$$




\subsection{Ascertainment of the Principal Result}

We substitute the solution $\mathrm{u}(\mathrm{z})$ of the form (8) in (6) and (7). For this purpose, we find:

$$
\begin{gathered}
\frac{\partial^{3} u}{\partial z^{3}}=\Phi_{1}^{\prime \prime \prime}\left(z+\mu_{1} \bar{z}\right)+\Phi_{2}^{\prime \prime \prime}\left(z+\mu_{2} \bar{z}\right)+\Phi_{3}^{\prime \prime \prime}\left(z+\mu_{3} \bar{z}\right)+\mu_{4}^{3} \Phi_{4}^{\prime \prime \prime}\left(\bar{z}+\mu_{4} z\right), \\
\frac{\partial^{3} u}{\partial z^{2} \partial \bar{z}}=\mu_{1} \Phi_{1}^{\prime \prime \prime}\left(z+\mu_{1} \bar{z}\right)+\mu_{2} \Phi_{2}^{\prime \prime \prime}\left(z+\mu_{2} \bar{z}\right)+\mu_{3} \Phi_{3}^{\prime \prime \prime}\left(z+\mu_{3} \bar{z}\right)+\mu_{4}^{2} \Phi_{4}^{\prime \prime \prime}\left(\bar{z}+\mu_{4} z\right), \\
\frac{\partial^{3} u}{\partial z \partial \bar{z}^{2}}=\mu_{1}^{2} \Phi_{1}^{\prime \prime \prime}\left(z+\mu_{1} \bar{z}\right)+\mu_{2}^{2} \Phi_{2}^{\prime \prime \prime}\left(z+\mu_{2} \bar{z}\right)+\mu_{3}^{2} \Phi_{3}^{\prime \prime \prime}\left(z+\mu_{3} \bar{z}\right)+\mu_{4} \Phi_{4}^{\prime \prime \prime}\left(\bar{z}+\mu_{4} z\right), \\
\frac{\partial^{2} u}{\partial z^{2}}=\Phi_{1}^{\prime \prime}\left(z+\mu_{1} \bar{z}\right)+\Phi_{2}^{\prime \prime}\left(z+\mu_{2} \bar{z}\right)+\Phi_{3}^{\prime \prime}\left(z+\mu_{3} \bar{z}\right)+\mu_{4}^{2} \Phi_{4}^{\prime \prime}\left(\bar{z}+\mu_{4} z\right), \\
\frac{\partial^{2} u}{\partial z \partial \bar{z}}=\mu_{1} \Phi_{1}^{\prime \prime}\left(z+\mu_{1} \bar{z}\right)+\mu_{2} \Phi_{2}^{\prime \prime}\left(z+\mu_{2} \bar{z}\right)+\mu_{3} \Phi_{3}^{\prime \prime}\left(z+\mu_{3} \bar{z}\right)+\mu_{4} \Phi_{4}^{\prime \prime}\left(\bar{z}+\mu_{4} z\right), \\
\frac{\partial^{3} u}{\partial \bar{z}^{3}}=\mu_{1}^{3} \Phi_{1}^{\prime \prime \prime}\left(z+\mu_{1} \bar{z}\right)+\mu_{2}^{3} \Phi_{2}^{\prime \prime \prime}\left(z+\mu_{2} \bar{z}\right)+\mu_{3}^{3} \Phi_{3}^{\prime \prime \prime}\left(z+\mu_{3} \bar{z}\right)+\Phi_{4}^{\prime \prime \prime}\left(\bar{z}+\mu_{4} z\right), \\
\frac{\partial^{2} u}{\partial \bar{z}^{2}}=\mu_{1}^{2} \Phi_{1}^{\prime \prime}\left(z+\mu_{1} \bar{z}\right)+\mu_{2}^{2} \Phi_{2}^{\prime \prime}\left(z+\mu_{2} \bar{z}\right)+\mu_{3}^{2} \Phi_{3}^{\prime \prime}\left(z+\mu_{3} \bar{z}\right)+\Phi_{4}^{\prime \prime}\left(\bar{z}+\mu_{4} z\right)
\end{gathered}
$$

Further, we expand the right-hand sides $F_{1}(z)$ and $F_{2}(z)$ of boundary conditions in series on the boundary $\partial K$ and denote their coefficients by $F_{ \pm k}$ and $G_{ \pm k}$, respectively: $F_{1}(z)=\sum_{k=1}^{\infty} F_{k} z^{k}+\sum_{k=1}^{\infty} F_{-k} \bar{z}^{k}, F_{2}(z)=$ $\sum_{k=1}^{\infty} G_{k} z^{k}+\sum_{k=1}^{\infty} G_{-k} \bar{z}^{k}$.

Then we obtain from the first boundary condition (6):

$$
\begin{gathered}
z^{2}\left(\sum_{k=1}^{\infty} A_{k} k z^{k-1}+\sum_{k=1}^{\infty} A_{k} k\left(\mu_{1} \bar{z}\right)^{k-1}\right. \\
+\sum_{k=1}^{\infty} B_{k} k z^{k-1}+\sum_{k=1}^{\infty} B_{k} k\left(\mu_{2} \bar{z}\right)^{k-1}+\sum_{k=1}^{\infty} C_{k} k z^{k-1}
\end{gathered}
$$




$$
\begin{aligned}
& \left.+\sum_{k=1}^{\infty} C_{k} k\left(\mu_{3} \bar{z}\right)^{k-1}+\sum_{k=1}^{\infty} D_{k} k \mu_{4}^{3}(\bar{z})^{k-1}+\sum_{k=1}^{\infty} D_{k} k \mu_{4}^{3}\left(\mu_{4} z\right)^{k-1}\right) \\
& +2 z \bar{z}\left(\sum_{k=1}^{\infty} A_{k} k \mu_{1} z^{k-1}+\sum_{k=1}^{\infty} A_{k} k \mu_{1}\left(\mu_{1} \bar{z}\right)^{k-1}+\sum_{k=1}^{\infty} B_{k} \mu_{2} k z^{k-1}\right. \\
& +\sum_{k=1}^{\infty} B_{k} \mu_{2} k\left(\mu_{2} \bar{z}\right)^{k-1}+\sum_{k=1}^{\infty} C_{k} \mu_{3} k z^{k-1}+\sum_{k=1}^{\infty} C_{k} k \mu_{3}\left(\mu_{3} \bar{z}\right)^{k-1} \\
& \left.+\sum_{k=1}^{\infty} D_{k} k \mu_{4}^{2}(\bar{z})^{k-1}+\sum_{k=1}^{\infty} D_{k} k \mu_{4}^{2}\left(\mu_{4} z\right)^{k-1}\right)+2 \bar{z}\left(\sum_{k=1}^{\infty} A_{k} k \mu_{1}^{2} z^{k-1}\right. \\
& +\sum_{k=1}^{\infty} A_{k} k \mu_{1}^{2}\left(\mu_{1} \bar{z}\right)^{k-1}+\sum_{k=1}^{\infty} B_{k} \mu_{2}^{2} k z^{k-1}+\sum_{k=1}^{\infty} B_{k} \mu_{2}^{2} k\left(\mu_{2} \bar{z}\right)^{k-1} \\
& +\sum_{k=1}^{\infty} C_{k} \mu_{3}^{2} k z^{k-1}+\sum_{k=1}^{\infty} C_{k} k \mu_{3}^{2}\left(\mu_{3} \bar{z}\right)^{k-1}+\sum_{k=1}^{\infty} D_{k} k \mu_{4}(\bar{z})^{k-1} \\
& \left.+\sum_{k=1}^{\infty} D_{k} k \mu_{4}\left(\mu_{4} z\right)^{k-1}\right)+2 z\left(\sum_{k=1}^{\infty} A_{k} z^{k}+\sum_{k=1}^{\infty} A_{k}\left(\mu_{1} \bar{z}\right)^{k}\right. \\
& +\sum_{k=1}^{\infty} B_{k} z^{k}+\sum_{k=1}^{\infty} B_{k}\left(\mu_{2} \bar{z}\right)^{k} \\
& +\sum_{k=1}^{\infty} C_{k} z^{k}+\sum_{k=1}^{\infty} C_{k}\left(\mu_{3} \bar{z}\right)^{k}+\sum_{k=1}^{\infty} D_{k} \mu_{4}^{2}(\bar{z})^{k} \\
& \left.+\sum_{k=1}^{\infty} D_{k} \mu_{4}^{2}\left(\mu_{4} z\right)^{k}\right)+2 \bar{z}\left(\sum_{k=1}^{\infty} A_{k} \mu_{1} z^{k}+\sum_{k=1}^{\infty} A_{k} \mu_{1}\left(\mu_{1} \bar{z}\right)^{k}\right. \\
& +\sum_{k=1}^{\infty} B_{k} \mu_{2} z^{k}+\sum_{k=1}^{\infty} B_{k} \mu_{2}\left(\mu_{2} \bar{z}\right)^{k}+\sum_{k=1}^{\infty} C_{k} \mu_{3} z^{k} \\
& \left.+\sum_{k=1}^{\infty} C_{k} \mu_{3}\left(\mu_{3} \bar{z}\right)^{k}+\sum_{k=1}^{\infty} D_{k} \mu_{4}(\bar{z})^{k}+\sum_{k=1}^{\infty} D_{k} \mu_{4}\left(\mu_{4} z\right)^{k}\right) \\
& =\sum_{k=1}^{\infty} F_{k} z^{k}+\sum_{k=1}^{\infty} F_{-k} \bar{z}^{k} .
\end{aligned}
$$

The second boundary condition (7) yields 


$$
\begin{aligned}
& \bar{z}^{2}\left(\sum_{k=1}^{\infty} A_{k} \mu_{1}^{3} k z^{k-1}+\sum_{k=1}^{\infty} A_{k} k \mu_{1}^{3}\left(\mu_{1} \bar{z}\right)^{k-1}+\sum_{k=1}^{\infty} B_{k} \mu_{2}^{3} k z^{k-1}\right. \\
& +\sum_{k=1}^{\infty} B_{k} \mu_{2}^{3} k\left(\mu_{2} \bar{z}\right)^{k-1}+\sum_{k=1}^{\infty} C_{k} \mu_{3}^{3} k z^{k-1}+\sum_{k=1}^{\infty} C_{k} \mu_{3}^{3} k\left(\mu_{3} \bar{z}\right)^{k-1} \\
& \left.+\sum_{k=1}^{\infty} D_{k} k(\bar{z})^{k-1}+\sum_{k=1}^{\infty} D_{k} k\left(\mu_{4} z\right)^{k-1}\right)+2 z \bar{z}\left(\sum_{k=1}^{\infty} A_{k} k \mu_{1}^{2} z^{k-1}\right. \\
& +\sum_{k=1}^{\infty} A_{k} k \mu_{1}^{2}\left(\mu_{1} \bar{z}\right)^{k-1}+\sum_{k=1}^{\infty} B_{k} \mu_{2}^{2} k z^{k-1}+\sum_{k=1}^{\infty} B_{k} \mu_{2}^{2} k\left(\mu_{2} \bar{z}\right)^{k-1} \\
& +\sum_{k=1}^{\infty} C_{k} \mu_{3}^{2} k z^{k-1}+\sum_{k=1}^{\infty} C_{k} k \mu_{3}^{2}\left(\mu_{3} \bar{z}\right)^{k-1}+\sum_{k=1}^{\infty} D_{k} k \mu_{4}(\bar{z})^{k-1} \\
& \left.+\sum_{k=1}^{\infty} D_{k} k \mu_{4}\left(\mu_{4} z\right)^{k-1}\right)+z^{2}\left(\sum_{k=1}^{\infty} A_{k} k \mu_{1} z^{k-1}+\sum_{k=1}^{\infty} A_{k} k \mu_{1}\left(\mu_{1} \bar{z}\right)^{k-1}\right. \\
& +\sum_{k=1}^{\infty} B_{k} \mu_{2} k z^{k-1}+\sum_{k=1}^{\infty} B_{k} \mu_{2} k\left(\mu_{2} \bar{z}\right)^{k-1}+\sum_{k=1}^{\infty} C_{k} \mu_{3} k z^{k-1} \\
& \left.+\sum_{k=1}^{\infty} C_{k} k \mu_{3}\left(\mu_{3} \bar{z}\right)^{k-1}+\sum_{k=1}^{\infty} D_{k} k \mu_{4}^{2}(\bar{z})^{k-1}+\sum_{k=1}^{\infty} D_{k} k \mu_{4}^{2}\left(\mu_{4} z\right)^{k-1}\right) \\
& +2 \bar{z}\left(\sum_{k=1}^{\infty} A_{k} \mu_{1}^{2} z^{k}+\sum_{k=1}^{\infty} A_{k} \mu_{1}^{2}\left(\mu_{1} \bar{z}\right)^{k}+\sum_{k=1}^{\infty} B_{k} \mu_{2}^{2} z^{k}+\sum_{k=1}^{\infty} B_{k} \mu_{2}^{2}\left(\mu_{2} \bar{z}\right)^{k}\right. \\
& \left.+\sum_{k=1}^{\infty} C_{k} \mu_{3}^{2} z^{k}+\sum_{k=1}^{\infty} C_{k} \mu_{3}^{2}\left(\mu_{3} \bar{z}\right)^{k}+\sum_{k=1}^{\infty} D_{k}(\bar{z})^{k}+\sum_{k=1}^{\infty} D_{k}\left(\mu_{4} z\right)^{k}\right) \\
& +2 z\left(\sum_{k=1}^{\infty} A_{k} \mu_{1} z^{k}+\sum_{k=1}^{\infty} A_{k} \mu_{1}\left(\mu_{1} \bar{z}\right)^{k}+\sum_{k=1}^{\infty} B_{k} \mu_{2} z^{k}+\sum_{k=1}^{\infty} B_{k} \mu_{2}\left(\mu_{2} \bar{z}\right)^{k}\right. \\
& \left.+\sum_{k=1}^{\infty} C_{k} \mu_{3} z^{k}+\sum_{k=1}^{\infty} C_{k} \mu_{3}\left(\mu_{3} \bar{z}\right)^{k}+\sum_{k=1}^{\infty} D_{k} \mu_{4}(\bar{z})^{k}+\sum_{k=1}^{\infty} D_{k} \mu_{4}\left(\mu_{4} z\right)^{k}\right) \\
& =\sum_{k=1}^{\infty} G_{k} z^{k}+\sum_{k=1}^{\infty} G_{-k} \bar{z}^{k}
\end{aligned}
$$


Using these two previous conditions, we arrive at four equations (two equations from the first condition above and, correspondingly, two equations from the second condition above) for the coefficients of expansion of the unknown functions (8):

$$
\begin{gathered}
A_{k-1}(k+1)+2 A_{k+1}(k+2) \mu_{1}+A_{k+3}(k+3) \mu_{1}^{2}+B_{k-1}(k+1) \\
+2 B_{k+1}(k+2) \mu_{2}+B_{k+3}(k+3) \mu_{2}^{2}+C_{k-1}(k+1)+2 C_{k+1}(k+2) \mu_{3} \\
+C_{k+3}(k+3) \mu_{3}^{2}+D_{k-1}(k+1) \mu_{4}^{k+1}+2 D_{k+1} \mu_{4}^{k+2}(k+2) \\
+D_{k+3} \mu_{4}^{k+3}(k+3)=F_{k},
\end{gathered}
$$

$$
\begin{gathered}
A_{k-1} \mu_{1}^{k}(k+1)+2 A_{k+1} \mu_{1}^{k+1}(k+2)+A_{k+3} \mu_{1}^{k+2}(k+3)+B_{k-1} \mu_{2}^{k}(k+1) \\
+2 B_{k+1} \mu_{2}^{k+1}(k+2)+B_{k+3}(k+3) \mu_{2}^{k+2}+C_{k-1} \mu_{1}^{k}(k+1)+2 C_{k+1} \mu_{1}^{k+1}(k+2) \\
+C_{k+3}(k+3) \mu_{3}^{k+2}+D_{k-1}(k+1) \mu_{4}+2 D_{k+1} \mu_{4}^{2}(k+2) \\
+D_{k+3} \mu_{4}^{3}(k+3)=F_{-k},
\end{gathered}
$$

$$
\begin{gathered}
A_{k-1} \mu_{1}(k+1)+2 A_{k+1} \mu_{1}^{2}(k+2) \mu_{1}+A_{k+3} \mu_{1}^{3}(k+3) \mu_{1}^{2}+B_{k-1} \mu_{2}(k+1) \\
+2 B_{k+1} \mu_{2}^{2}(k+2) \mu_{2}+B_{k+3} \mu_{3}^{3}(k+3) \mu_{2}^{2}+C_{k-1} \mu_{3}(k+1) \\
+2 C_{k+1} \mu_{3}^{2}(k+2) \mu_{3}+C_{k+3} \mu_{3}^{3}(k+3) \mu_{3}^{2}+D_{k-1}(k+1) \mu_{4}^{k} \\
\quad+2 D_{k+1} \mu_{4}^{k+1}(k+2)+D_{k+3} \mu_{4}^{k+2}(k+3)=G_{k},
\end{gathered}
$$

$$
\begin{gathered}
A_{k-1} \mu_{1}^{k+1}(k+1)+2 A_{k+1} \mu_{1}^{k+2}(k+2)+A_{k+3} \mu_{1}^{k+3}(k+3) \\
+B_{k-1} \mu_{2}^{k+1}(k+1)+2 B_{k+1} \mu_{2}^{k+2}(k+2)+B_{k+3}(k+3) \mu_{2}^{k+3} \\
+C_{k-1} \mu_{1}^{k+1}(k+1)+2 C_{k+1} \mu_{1}^{k+2}(k+2)+C_{k+3}(k+3) \mu_{3}^{k+3}+D_{k-1}(k+1) \\
+2 D_{k+1} \mu_{4}(k+2)+D_{k+3} \mu_{4}^{2}(k+3)=G_{-k} .
\end{gathered}
$$

To investigate further the system of recursive equations (11)-(14), we introduce the following auxiliary sequences of vectors:

$$
M_{k}=\left(A_{k}, B_{k}, C_{k}, D_{k}\right)^{T}, S_{k}=\left(F_{k}, F_{-k}, G_{k}, G_{-k}\right)^{T} .
$$

Then we may represent system (11)-(14) in the following matrix form

$$
\Delta_{k}\left(M_{k-1}+2 \mu_{1} \mu_{2} \mu_{3} \mu_{4} M_{k+1}+\mu_{1}^{2} \mu_{2}^{2} \mu_{3}^{2} \mu_{4}^{2} M_{k+3}\right)=S_{k},
$$


here

$$
\Delta_{k}=\left(\begin{array}{cccc}
1 & 1 & 1 & \mu_{4}^{k+1} \\
\mu_{1} & \mu_{2} & \mu_{3} & \mu_{4}^{k} \\
\mu_{1}^{k} & \mu_{2}^{k} & \mu_{3}^{k} & \mu_{4} \\
\mu_{1}^{k+1} & \mu_{2}^{k+1} & \mu_{3}^{k+1} & 1
\end{array}\right)
$$

Thus, the sufficient condition of solvability of Eq. (15) and, hence, the Neumann problem (1), (2) is

$$
\operatorname{det} \Delta_{\mathrm{k}} \neq 0, \forall \mathrm{k}
$$

Note that a similar condition arose in paper [5], where conditions of the existence and uniqueness of solution of the Dirichlet problem for improperly elliptic Eq. (1) with condition (3) were investigated. The solution of (15) exists because, according to (17), there exists $\Delta_{k}^{-1}, \forall k$. Performing the substitution

$$
T_{k}=\Delta_{k}^{-1} \cdot S_{k}, \quad \delta_{k}=\mu_{1} \mu_{2} \mu_{3} \mu_{4},
$$

we rewrite Eq. (15) in the form

$$
M_{k-1}+2 \delta M_{k+1}+\delta^{2} M_{k+3}=T_{k}
$$

The obtained vector equation (18) is a fourth-order recursive equation with constant coefficients (see [12]). Its solution $M_{k}$, according to the Moivre formulas [12], has the form

$$
\begin{aligned}
M_{k}=Q_{1}(k-1) & \left(\frac{i}{\delta^{1 / 2}}\right)^{k}+k Q_{2}(k-1)\left(\frac{i}{\delta^{1 / 2}}\right)^{k}+Q_{3}(k-1)\left(\frac{-i}{\delta^{1 / 2}}\right)^{k} \\
& +k Q_{4}(k-1)\left(\frac{-i}{\delta^{1 / 2}}\right)^{k}+F\left(\Delta_{k}^{-1} \cdot S_{k}\right)
\end{aligned}
$$

where $Q_{j}(k-1), j=1, \ldots, 4$, are certain vector polynomials and $F$ is function, depending from right-hand part $S_{k}$. Hence, we have established the sufficient conditions of existence of the analytic functions (8) (formulas for the sequences $M_{k}=\left(A_{k}, B_{k}, C_{k}, D_{k}\right)^{T}$ of coefficients $A_{k}, B_{k}, C_{k}, D_{k}, k=1,2, \ldots$, of the expansions of these functions have been constructed) and, therefore, of the existence of a solution of the Neumann problem (1), (2) in the form (8). Thus, we have proved the following theorem of sufficient conditions of the solvability of the Neumann problem (1), (2) for fourth-order improperly elliptic equations with the condition (3).

Now, let us study the asymptotic behavior of the determinant of matrix (16) for sufficiently large k. Let $\left|\mu_{1}\right|>\left|\mu_{2}\right|>\left|\mu_{3}\right|$ and $\alpha_{1}=\frac{\mu_{2}}{\mu_{1}}, \beta_{1}=$ 
$\frac{\mu_{3}}{\mu_{1}}, \gamma_{1}=\mu_{1} \mu_{4}$. Then, by virtue of $(3),\left|\alpha_{1}\right|<1,\left|\beta_{1}\right|<1$, and $\left|\gamma_{1}\right|<1$, we have

$$
\operatorname{det} \Delta_{\mathrm{k}}=\mu_{1}^{\mathrm{k}+1} \operatorname{det}\left(\begin{array}{cccc}
1 & 1 & 1 & \gamma^{k+1} \\
1 & \alpha_{1} & \beta_{1} & \gamma_{1}^{k} \\
1 & \alpha_{1}^{k} & \beta_{1}^{k} & \gamma_{1} \\
1 & \alpha_{1}^{k+1} & \beta_{1}^{k+1} & 1
\end{array}\right) \rightarrow\left(1-\gamma_{1}\right)\left(\beta_{1}-\alpha_{1}\right) \neq 0 .
$$

The fact that condition (17) does not hold for at least some $\mathrm{k}$ (i.e., the kernel is nontrivial) is illustrated by the following example. In (19), let $k=3, \mu_{1}=2 / 3, \mu_{2}=-1 / 2, \mu_{3}=0$ and $\mu_{4}=1 / 2$, i.e., $\alpha_{1}=-3 / 4, \beta_{1}=$ 0 , and $\gamma_{1}=1 / 3$. Then $\operatorname{det} \Delta_{4}=0$. Thus, for improperly elliptic equations satisfying condition (3), condition (17) does not satisfied only for finitely many $\mathrm{k}$ (so, condition (17) is true almost everywhere); consequently, in this case, the kernel of the Neumann problem (1), (2) is finite- dimensional and has

$$
0 \neq d=\sum_{k=4}^{\infty}\left(4-\operatorname{rank} \Delta_{k}\right)
$$

linearly independent elements (for the Dirichlet problem these elements have built in paper [6, see lemma 1]). Such a way we are coming to

Theorem 1. [13] Suppose that Eq. (1) is improperly elliptic, and $\lambda_{1}, \lambda_{2}$, $\lambda_{3}, \lambda_{4}$ are the roots of corresponding characteristic equation satisfying the conditions

$$
\operatorname{Im} \lambda_{1}>0, \operatorname{Im} \lambda_{2}>0, \operatorname{Im} \lambda_{3}>0, \operatorname{Im} \lambda_{4}<0,
$$

and $\lambda_{k} \neq \lambda_{j}$, for $k \neq j$, and $\lambda_{j} \neq \pm i, \forall j=1, \ldots, 4$. Let also

$$
\mu_{1}=-\frac{\lambda_{1}-i}{\lambda_{1}+i}, \mu_{2}=-\frac{\lambda_{2}-i}{\lambda_{2}+i}, \mu_{3}=-\frac{\lambda_{3}-i}{\lambda_{3}+i}, \mu_{4}=-\frac{\lambda_{4}+i}{\lambda_{4}-i} .
$$

satisfy the condition (16) and (17):

$$
\operatorname{det} \Delta_{\mathrm{k}}=\left(\begin{array}{cccc}
1 & 1 & 1 & \mu_{4}^{k+1} \\
\mu_{1} & \mu_{2} & \mu_{3} & \mu_{4}^{k} \\
\mu_{1}^{k} & \mu_{2}^{k} & \mu_{3}^{k} & \mu_{4} \\
\mu_{1}^{k+1} & \mu_{2}^{k+1} & \mu_{3}^{k+1} & 1
\end{array}\right) \neq 0
$$

Suppose also that the functions $f_{1} \in C^{1, \alpha}(\partial K)$ and $f_{2} \in C^{\alpha}(\partial K)$, $0<\alpha<1$, from conditions (2) are specified on the boundary $\partial K$ and can be prolonged to analytic functions in the disk $K$ and outside it. Then the Neumann problem (1), (2) will have a solution in the space $C^{4}(K) \cap$ $C^{3, \alpha}(\bar{K})$, which can be represented as

$$
u(z)=\Phi_{1}\left(z+\mu_{1} \bar{z}\right)+\Phi_{2}\left(z+\mu_{2} \bar{z}\right)+\Phi_{3}\left(z+\mu_{3} \bar{z}\right)+\Phi_{4}\left(\bar{z}+\mu_{4} z\right),
$$


where $\Phi_{k}, k=1,2,3,4$ are certain functions fairly smooth and analytic together with their derivatives and the column vector $M_{k}=\left(A_{k}, B_{k}, C_{k}, D_{k}\right)^{T}$ of coefficients $A_{k}, B_{k}, C_{k}, D_{k}, k=1,2, \ldots$ of the expansion of functions $\Phi_{k}, k=1,2,3,4$ is determined by the Moivre formulas

$$
\begin{gathered}
M_{k}=Q_{1}(k-1)\left(\frac{i}{\delta^{1 / 2}}\right)^{k}+k Q_{2}(k-1)\left(\frac{i}{\delta^{1 / 2}}\right)^{k} \\
+Q_{3}(k-1)\left(\frac{-i}{\delta^{1 / 2}}\right)^{k}+k Q_{4}(k-1)\left(\frac{-i}{\delta^{1 / 2}}\right)^{k}+F\left(\Delta_{k}^{-1} \cdot S_{k}\right),
\end{gathered}
$$

with certain vector polynomials $Q_{j}(k-1), j=1, \ldots, 4$ and function $F$, depending from right-hand part $S_{k}$.

Moreover, conditions (20) hold almost everywhere and the kernel of the Neumann problem (1), (2) is finite-dimensional and has

$$
0 \neq d=\sum_{k=4}^{\infty}\left(4-\operatorname{rank} \Delta_{k}\right),
$$

linearly independent elements.

\subsection{Improperly Elliptic Equations Satisfying Condition (4)}

Consider the complex numbers $\mu_{k}$ defined on the basis of the roots $\lambda_{k}, k=1, \ldots, 4$, of the characteristic equation by the formulas:

$$
\mu_{1}=-\frac{\lambda_{1}-i}{\lambda_{1}+i}, \mu_{2}=-\frac{\lambda_{2}-i}{\lambda_{2}+i}, \mu_{3}=-\frac{\lambda_{3}-i}{\lambda_{3}+i}, \mu_{4}=-\frac{\lambda_{4}-i}{\lambda_{4}+i} .
$$

In this case Eq. (5) will has the form:

$$
\left(\frac{\partial}{\partial z}-\mu_{1} \frac{\partial}{\partial \bar{z}}\right)\left(\frac{\partial}{\partial z}-\mu_{2} \frac{\partial}{\partial \bar{z}}\right)\left(\frac{\partial}{\partial z}-\mu_{3} \frac{\partial}{\partial \bar{z}}\right)\left(\frac{\partial}{\partial z}-\mu_{4} \frac{\partial}{\partial \bar{z}}\right) u(z)=0
$$

The solution $u(z)$ of Eq. (22) can be represented by the formula:

$$
u(z)=\Phi_{1}\left(z+\mu_{1} \bar{z}\right)+\Phi_{2}\left(z+\mu_{2} \bar{z}\right)+\Phi_{3}\left(z+\mu_{3} \bar{z}\right)+\Phi_{4}\left(z+\mu_{2} \bar{z}\right),
$$

where $\Phi_{k}, k=1,2,3,4$ are certain functions fairly smooth and analytic together with their derivatives. We find them from the boundary conditions (6), (7) with the help of lemma 1. Further, we use the technique, proposed above for the class (3) of improperly elliptic equations, and arrive at to the system of recursive equations in matrix form:

$$
\Delta_{k}\left(M_{k-1}+2 \mu_{1} \mu_{2} \mu_{3} \mu_{4} M_{k+1}+\mu_{1}^{2} \mu_{2}^{2} \mu_{3}^{2} \mu_{4}^{2} M_{k+3}\right)=S_{k} .
$$


This system is analog Eq. (15) but matrix $\Delta_{k}$ has another form:

$$
\Delta_{n}=\operatorname{det}\left(\begin{array}{cccc}
1 & 1 & 1 & 1 \\
\mu_{1} & \mu_{2} & \mu_{3} & \mu_{4} \\
\mu_{1}^{n-1} & \mu_{2}^{n-1} & \mu_{3}^{n-1} & \mu_{4}^{n-1} \\
\mu_{1}^{n} & \mu_{2}^{n} & \mu_{3}^{n} & \mu_{4}^{n}
\end{array}\right), \mathrm{n}>3 .
$$

Let $\left|\mu_{1}\right|>\left|\mu_{2}\right|>\left|\mu_{3}\right|>\left|\mu_{4}\right|, \alpha=\frac{\mu_{2}}{\mu_{1}}, \beta=\frac{\mu_{3}}{\mu_{1}}, \gamma=\frac{\mu_{4}}{\mu_{1}}$, and, in addition, $|\alpha|<1,|\beta|<1,|\gamma|<1$. Let us study the asymptotic behavior of the determinant (25) for sufficiently large $n$ :

$$
\Delta_{n}=\mu_{1}^{n} \operatorname{det}\left(\begin{array}{cccc}
1 & 1 & 1 & 1 \\
1 & \alpha & \beta & \gamma \\
1 & \alpha^{n-1} & \beta^{n-1} & \gamma^{n-1} \\
1 & \alpha^{n} & \beta^{n} & \gamma^{n}
\end{array}\right) \sim 0 .
$$

In the paper [6] there has been proved the following result.

Lemma 2. [6] Let Eq. (1) be elliptic but improperly elliptic with condition (4) for the roots. Then the corresponding Dirichlet problem has countably many linearly independent polynomial solutions.

Thus, for improperly elliptic equations satisfying condition (4), the kernel of the corresponding Dirichlet problem (so that the kernel of the Neumann problem (1), (2)) is infinite-dimensional and can be described with the use of polynomials of the form (23).

From formula (26) and lemma 2 we are coming to the conclusion that there does not exist $\Delta_{n}^{-1}$, so, the equation (24) is not solvable and corresponding Neumann problem is not Fredholm problem (or it is irregular problem).

Theorem 2. Suppose that Eq. (1) is improperly elliptic, and $\lambda_{1}, \lambda_{2}, \lambda_{3}$, $\lambda_{4}$ are the roots of corresponding characteristic equation satisfying the conditions

$$
\operatorname{Im} \lambda_{1}>0, \operatorname{Im} \lambda_{2}>0, \operatorname{Im} \lambda_{3}>0, \operatorname{Im} \lambda_{4}>0
$$

and $\lambda_{k} \neq \lambda_{j}$, for $k \neq j$, and $\lambda_{j} \neq \pm i, \forall j=1, \ldots, 4$. Let also

$$
\mu_{1}=-\frac{\lambda_{1}-i}{\lambda_{1}+i}, \mu_{2}=-\frac{\lambda_{2}-i}{\lambda_{2}+i}, \mu_{3}=-\frac{\lambda_{3}-i}{\lambda_{3}+i}, \mu_{4}=-\frac{\lambda_{4}-i}{\lambda_{4}+i} .
$$

Then the Neumann problem (1), (2) has infinite-dimensional kernel and it is not Fredholm problem. 


\section{Conclusions}

In the present paper it has been investigated the Neumann problem in a disk for all classes of improperly elliptic fourth order differential equation in general case, that is roots $\lambda_{1}, \lambda_{2}, \lambda_{3}, \lambda_{4}$ are different, do not equal $\pm i$, and satisfy conditions:

$$
\begin{aligned}
& \operatorname{Im} \lambda_{1}>0, \operatorname{Im} \lambda_{2}>0, \operatorname{Im} \lambda_{3}>0, \operatorname{Im} \lambda_{4}<0, \\
& \operatorname{Im} \lambda_{1}>0, \operatorname{Im} \lambda_{2}>0, \operatorname{Im} \lambda_{3}>0, \operatorname{Im} \lambda_{4}>0 .
\end{aligned}
$$

We expanded the methods of investigations of our previous works $[6,7]$, which were applied for studying the Dirichlet and properly elliptic Neumann problems for the case of improperly elliptic Neumann problem. In this paper we have obtained sufficient condition for existence of solution of posed Neumann problem in the first case of improperly elliptic equations (which satisfy conditions (3)) and we have proved that in the second case of improperly elliptic equations (which satisfy conditions (4)) correspondent Neumann problem (1), (2) is irregular problem.

\section{Acknowledgments}

This work is supported by grants of Ministry of Education and Science of Ukraine (project numbers are 0115U000136, 0116U004691).

\section{References}

[1] G. Grubb Distributions and Operators (Graduate Texts in Mathematics), Springer, New York, 2009.

[2] L. Hormander The Analysis of Linear Partial Differential Operators I. Distribution Theory and Fourier Analysis, Springer, Berlin, 1983.

[3] A. V. Bitsadze, On solution uniqueness of the Dirichlet problem for partial elliptic equations // Uspehi Mat. Nauk, 3 (1948), 211-212.

[4] S. Albeverio, F. Gesztesy, R. Hoegh-Krohn, and H. Holden, Solvable Models in Quantum Mechanics, Springer, Berlin, 1988.

[5] B. Brown, G. Grubb, and I. G. Wood, M-functions for closed extensions of adjoint pairs of operators with applications to elliptic boundary problems // Math. Nachr, 282 (2009), 314-347.

[6] E. A. Buryachenko, On the dimension of kernel of the Dirichlet problem for fourth order equations // Differential Equations, 51 (2015), No. 4, 1-10.

[7] K. O. Buryachenko, A. I. Harabara, Solvability of the Neumann problem in a disk for fourth order properly elliptic equation // Journal of Mathematical Sciences, 212 (2016), No. 1, 1-10. 
[8] K. O. Buryachenko, Solvability of inhomogeneous boundary-value problems for fourth-order differential equations // Ukrainian Math. Journal, 63 (2012), No. 8, $1165-1175$.

[9] G. Bonanno, P. F. Pizzimenti, Neumann boundary value problems with not coercive potential // Mediterr. J. Math., 9 (2012), No. 4, 601-609.

[10] V. P. Burskii, K. A. Buryachenko, On the breakdown of the uniqueness of a solution of the Dirichlet problem for typeless differential equations of arbitrary even order in a disk // J. Math. Sci., 190 (2013), No. 4, 539-566.

[11] N. E. Tovmasyan, Non-Regular Differential Equations and Calculations of Electromagnetic Fields, World Scientific Publ., Singapore, 1998.

[12] Jay P. Fillmore, Morris L. Marx, Linear recursive sequences // SIAM Rev. (1968), 324-353.

[13] K. O. Buryachenko, Solvability of the Neumann problem for some class of improperly elliptic fourth-order equations // Cherkasy University Bulletin: Physical and Mathematical Sciences, 349 (2015), No. 16, 16-23.

\section{CONTACT INFORMATION}

Kateryna

Olexandrivna

Buryachenko

Yuliya Kudrych
Vasyl' Stus Donetsk National University,

Vinnytsia, Ukraine

E-Mail: katarzyna_@ukr.net

Vasyl' Stus Donetsk National University,

Vinnytsia, Ukraine

E-Mail: uliakudrych1994@gmail.com 